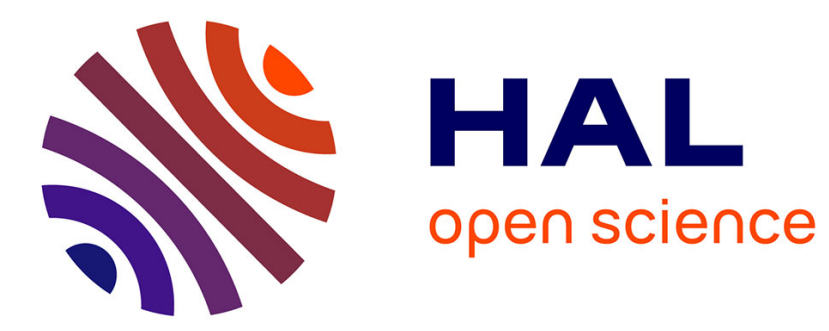

\title{
New strategy for resource allocation using PSO-PFS hybrid
}

\author{
Zenadji Sylia, Cédric Gueguen, Brikh Lamine, Talbi Larbi, Khireddine
}

Abdelkrim

\section{To cite this version:}

Zenadji Sylia, Cédric Gueguen, Brikh Lamine, Talbi Larbi, Khireddine Abdelkrim. New strategy for resource allocation using PSO-PFS hybrid. International Journal of Wireless and Mobile Computing, 2020. hal-03124029

\author{
HAL Id: hal-03124029 \\ https://hal.science/hal-03124029
}

Submitted on 28 Jan 2021

HAL is a multi-disciplinary open access archive for the deposit and dissemination of scientific research documents, whether they are published or not. The documents may come from teaching and research institutions in France or abroad, or from public or private research centers.
L'archive ouverte pluridisciplinaire HAL, est destinée au dépôt et à la diffusion de documents scientifiques de niveau recherche, publiés ou non, émanant des établissements d'enseignement et de recherche français ou étrangers, des laboratoires publics ou privés. 


\title{
New strategy for resource allocation using PSO-PFS hybrid
}

\author{
Zenadji Sylia ${ }^{1}$, Gueguen Cédric ${ }^{2}$, Brikh Lamine ${ }^{3}$, Talbi Larbi ${ }^{4}$, Khireddine Abdelkrim $^{1}$ \\ ${ }^{1}$ Laboratoire de Génie Electrique (LGEB) \\ Faculté de Technologie, Université de Bejaia, 06000 Bejaia, Algeria \\ zenadji.sylia@gmail.com \\ abdelkrim.khired@gmail.com \\ ${ }^{2}$ Laboratoire IRISA \\ Université de Beaulieu Rennes1, 35042 Rennes, France \\ cedric.gueguen@irisa.fr \\ ${ }^{3}$ Laboratoire de Technologie Industrielle et de l'information \\ Faculté de Technologie, Université de Bejaia, 06000 Bejaia, Algeria \\ blamine@outlook.fr \\ ${ }^{4}$ Laboratoire de recherche sur les communications sans fil large bande (LRCL) \\ Université du Québec en Outaouais J8X 3X7, Gatineau (Québec) Canada \\ Larbi.Talbi@uqo.ca
}

\begin{abstract}
In this article, we study the problem of resource allocation in a tri-sectoral cell in order to have an equal distribution of resources among users taking into account the OFDM transmission technique that has evolved in future wireless networks LTE and 5G. We propose a new strategy for resource allocation schemes able to help the users with a critical position thanks to hybridization PSO-PFS (Particle Swarm Optimization). In addition, PFS (Proportional Fair Scheduling) it take into consideration the channel state conditions of the users and the PSO algorithm provides an optimal solution to the allocation problem and improves the performances of users according to their stat. The simulation results show that the allocation of resources by the PSO-PFS hybrid algorithm guarantee a high throughput of the system by ensuring fairness between users.
\end{abstract}

Keywords: Resource Allocation; 5G; Tri-sectoral Cell; PSO-PFS; Throughput; Fairness.

1. Introduction

Ensuring a real quality of service QoS in mobile multimedia transmissions is an important challenge. One of the major features of wireless networks are subject to many channel impairments (pathloss, shadowfading, multipath fading) [1]. These phenomena severely affect the quality of service in terms of delays or packet loss, in this case the users can not benefit from the same transmission capacity. Therefore at some point a radio resource may be of good quality for one user as it may be bad for another. In this context, in recent years, researchers have paid considerable attention to the allocation of resources for digital communication networks. Among all the transmission techniques studied, Orthogonal Frequency Division Multiplexing OFDM has emerged widely for new generation transmission networks, LTE and 5G [2]. On the other hand, the OFDM is an access method that allows the bandwidth to be divided into several sub-frequency bands. In addition, each carrier has a low bandwidth in relation to the total bandwidth used, which leads to a high tolerance for multipath propagation problems. In [3], the authors provide 
an equitable distribution of resources to fight against the impact of multipath propagation, but in return, the throughput of the system is reduced. The authors of [4] proposed a resource allocation based on orthogonal frequency division multiplexing (OFDMA), assuming a complete reuse of the frequency between macrocells and microcells.

Various works have generated great interest for new Scheduling and Optimization algorithms. In the first, the opportunistic Scheduling algorithms attribute a higher priority to users with better channel conditions [5]. The research in [6] evaluate different strategies of resource allocation from different schedulers and conclude that performance comprehensively depend upon channel imperfection. The inter-cellular scheduler algorithms control the resource allocation and allow intelligent distribution of the subcarriers in a cellular network. In [2] propose a new intercellular Bandwidth Fair Sharing Scheduler (IBFS) able to help the overloaded cells thanks to a dynamic cell bandwidth allocation in multi-user $5 \mathrm{G}$ wireless networks. FFRDIBFS is proposed by [7] which distribute the frequencies in the overloaded cells without destroying the QOS of the underloaded cells, where the objective is to dynamically calculate the minimum bandwidth of an underloaded cell. In the second, the major advantage of using the evolutionary algorithms is the maximum exploitation and exploration of the search space to find the global optimum solution or near global optimum solution. In [8] a genetic algorithm is proposed for improving efficient resource allocation Macrocell-femtocell, the proposed model encourages femtocell owners to share resources with public users. A dynamic optimization of resources based on the Particle Swarm Optimization algorithm (PSO) considering the fading channel, the change of channel state of the users and the relay stations in a macro-cellular [9] has been suggested to achieve better performance in terms of throughput and equitability. The resource allocation based on PSO (Particle Swarm Optimization) and hybrid PSOGA (Genetic Algorithm) [10] have been proposed to maximize the throughput of the two-pair D2D communication system of the whole macro cell.

Although the quality of service (QoS) of intracellular wireless networks has improved significantly, recently several studies have been proposed studying the sectoral spectrum allocation mechanism, which improves system performance. In [11] a new frequency reuse scheme is proposed, the sub-carriers are divided into two groups, in the central region of the sectors, and at the edge of the three sectors. A spectrum distribution into four sub-bands and allocating those to different sectors with six directional antennas macrocell to reduce interference has been studied in [12]; the results show that the utilizing six-sectors, the throughput of macrocells progressively increases. In [13] authors have proposed a sector offset configuration strategy for multi-carrier networks, the pattern offset is 60 degrees.

QoS cannot be guaranteed when the users are located in critical positions. The position of users has a direct relationship to channel conditions, and the constraint of induced path losses has an unequal spectral efficiency, typically for users in regions of sectoral overlap, causing cross-sectoral interference and a low SINR. However, for such a constraint implies an inequality in terms of throughput and fairness between different users. 5G systems should take over the design of techniques that effectively manage all spectral resources; in addition, these main objectives are to provide high throughput in the cell with low latency [14]. The opportunistic Scheduling favors users with better channel conditions and the major concern for the scheduling discipline should be to maximize the total throughput for all users. In this context, to resolve this problem, the contributions of this paper are summarized as follows. First, we exploit frequencies in an opportunistic way, do not 
make a 50/50 frequency distribution in each sector of the cell but adapt it according to the position of the users and their requests. Second, in order to overcome the limitation of the Scheduler Proportional Fair in terms of throughput, we propose a hybrid PSO-PFS that can improve the performance of PFS in a tri-sectoral cell, we present and explain the concept of resource allocation in a Tri-sectoral cell, which corresponds to the amount of radio resources that can be allocated while maintaining frequency reuse1.

The remainder of this work is organized as follows. System model is presented in section 2 . Scheduling algorithm strategies are given in section 3. Section 4 presents the performance evaluation results of our proposed resource allocation schemes. Finally, in section 5, we outline the conclusion of this paper and future work.

\section{System Model and Problem Description \\ 2.1.Channel Model}

We consider a tri-sectoral cell with a base station (BS) and $\mathrm{K}$ mobile stations (MS) uniformly distributed in the cell, An equal frequency distribution is applied, sharing a total number of $\mathrm{N}$ subcarriers in the cell (see figure.1).

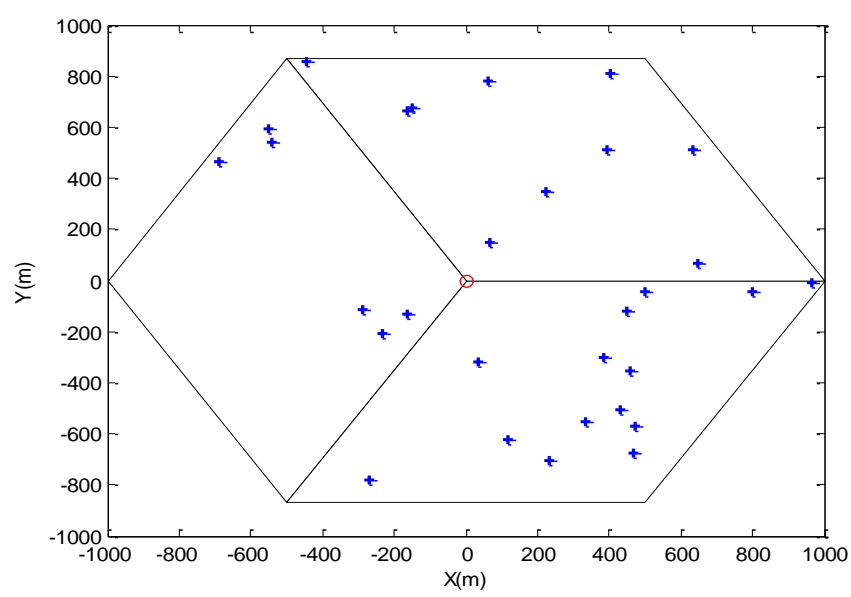

Figure 1 Location of the users in the cell.

In our scheme, we propose an allocation of resources according to the position of the users.
Note that in this way users with high interference between sectors and at the ends of the cell will benefit from the same quality of service as users with high SINR. The signal to interference and noise ratio (SINR) of user $\mathrm{k}$ on subcarrier $\mathrm{n}$ at time $t$ is given by:

$$
\operatorname{SINR}_{k, n}^{s}(t)=\frac{p_{k, n}^{s} G^{s} k, n}{\left(\sum_{m=1}^{M} p_{m, n}^{s} G^{s}{ }_{m, n}\right)+P_{N}}
$$

where $p^{s} k, n$ is the transmit power on the $n^{\text {th }}$ subcarrier to $k^{\text {th }}$ mobile in $S$ sector, $P_{N}$ is the noise power spectrum density Gaussian at each time slot, $p^{s} m, n$ is the power for user $k^{\text {th }}$ on the same subcarrier $m^{\text {th }}$, and $G_{k, n}^{s}$ is the channel gain, the users suffers from the Path loss, multipath fading and Shadow fading.

The overall channel gain is calculated as a sum of individuel gains :

$$
G_{k, n}^{s}(t)=G_{A} * G_{P} *\left|h_{k, n}(t)\right|^{2} * G_{S}
$$

The antenna gain of each BS sector is given by:

$$
G_{A}=G_{\max }+G_{\varphi}+G_{\theta}
$$

where $G_{\max }=15 \mathrm{dBi}$ is the maximum antenna gain and we applying the two formulas below for horizontal and vertical radiation patterns respectively $[15,16]$ :

$$
G \varphi=-\min \left(12\left(\frac{\varphi}{\alpha}\right)^{2}, A_{m}\right)
$$

where $\varphi\left(-180^{\circ} \leq \varphi \leq 180^{\circ}\right)$ is the azimuth angle, $\alpha=65^{\circ}$ (3sectors) is horizontal halfpower beamwidth, and $A_{m}=30 \mathrm{~dB}$ is the front-back attenuation.

$G_{\theta}=-\min \left(12\left(\frac{\theta}{\alpha}\right)^{2}, S L A_{v}\right)$

where $\theta\left(-15^{\circ} \leq \theta \leq 0^{\circ}\right)$ is the tilt angle, and $\alpha=10^{\circ}$ is vertical halfpower beam-width and $S L A_{v}=10^{\circ}$ is side lobe attenuation. 
The multipath fading channel $h_{k, n}(t)$ is modeled using Rayleigh fading, the channel is its time-varying, this time variation due to the movement of the mobile or objects within the channel [17].

The propagation path loss $G_{P}=\left(d_{0} / d_{k}^{s}\right)^{\alpha}$ dependent on the $d_{0}=1 \mathrm{Km}$ is the reference distance, $\alpha$ is the path loss exponent, for the location of the user $k$ in the sector $S$, we calculate the distance $d_{k}^{s}$ is given by:

$d_{k}^{s}=\sqrt{\left(x_{s}-x_{k}\right)^{2}+\left(y_{s}-y_{k}\right)^{2}}$

The shadow fading $G_{S}$ are generated with lognormal distribution and the random variations in signal amplitude follow a Gaussian distribution with mean of zero and standard deviation of $\sigma$ [18]. Sectors from the same base station site use the same shadow fading values [13].

The transmission rate of each $k^{\text {th }}$ user terminal is calculated as from the SINR, is got by [17]:

$$
r_{k, n}^{s}(t)=W \log _{2}\left(1+\operatorname{SINR}_{k, n}^{s}(t)\right)
$$

On the other hand the achievable rate of the cell can be given by:

$$
R_{k, n}^{s}\left(\operatorname{SINR}_{k, n}^{s}\right)=\sum_{s=1}^{3} \sum_{n=1}^{N} \rho_{k, n}^{s} r_{k, n}^{s}
$$

where $\operatorname{SINR}_{k, n}^{S}$ is the stat indication of users including the location of each user. $\rho_{k, n}^{s}=1$; indicates that the subcarrier $n^{\text {th }}$ is assigned to the user $k^{\text {th }}$; else $\rho_{k, n}^{s}=0$

\subsection{Problem Description}

In this section, we present a mathematical model that is used in this article. Compared to other proposed models, the optimization approach proposed has the advantages of simplicity, flexibility and high accuracy that can be applied to other scenarios. The proposed resource allocation scheme is formulated as follows:

$$
C=\left\{b_{1}, b_{2}, \ldots . . b_{N}\right\}, \text { where } b_{n} \in(1, K)
$$

$\mathrm{C}$ as the matrix of the resource assigned to the $b_{n}{ }^{t h}$ mobile in $n^{\text {th }}$ subcarrier during a time interval according to PF Scheduling.

In this scenario the optimization problems can be formulated as follows, our objective is to maximize the utility of all users, is defined as follow Problem:

$$
\delta=\max \mathrm{u}_{t}(\mathrm{C})
$$

Obviously $t$ is the index of time for different slot. On the other hand, to achieve our goal, constraints are added to the optimization problem of proposed resource allocation schemes, two constraints, namely

- The total transmit power assigned to users must be less than or equal to maximum transmitted power allowed $P_{\max }[19]$.

$$
\sum_{n=1}^{N} p_{k, n}^{s} \leq P_{\max } \forall k \in[1, K]
$$

- In our system, the number of active users can change position and also the channel state conditions of the users change, all these factors are contained in the SINR.

The System Throughput performance of all users is defined as follows

$$
S T=\frac{1}{T} \sum_{t=1}^{T}\left(k^{*}, \text { gbest }\right)
$$

The System Fairness performance as a function of the number of users is defined as:

$$
S F=-\sum_{k=1}^{K} C_{k} \frac{\log C_{k}}{\log K}
$$


where $C_{k}$ is the resources allocated to user $\mathrm{k}$ and the $\log K$ term is a normalized factor.

\section{Resource Allocation Algorithm}

Our contribution relies on the use of Hybridation PSO-PFS algorithm. We will give a preview on the scheduler Proportional Fair in section 3.1. After, the overview of the PSO Algorithm in section 3.2. In section 3.3 we develop the Hybridation PSOPFS Based Resource allocation algorithm in detail.

\subsection{PFS Algorithm}

The Scheduling is currently hosted in the MAC layer are based on opportunistic resource allocation. First appearance was proposed in [20]. The scheduler must efficiently allocate available bandwidth resources provided by the physical layer to maximize system performance [21], in terms of throughput, fairness and delay. A mobile with a high SINR has a high priority, compared to users with a low SINR. Since PFS is based on variation in channel conditions mainly due to multipath fading, all mobiles get the same probability of access to resources across time [22]. As such, this algorithm offers a tradeoff between throughput and fairness [23].

However, its principle is based on a kind of feedback between the base station and the users to obtain an estimation of the channel state for each user and the instantaneous data rate in each subcarrier. The PF scheduler assigns each resource on the subcarrier $n$ in $(t)$ slots, and then it monitors the average throughput in each subcarrier at each Transmit Time Interval (TTI) in a window of length $t c[24,25]$.

The objective function representing the $\mathrm{PF}$ Scheduling algorithm is $k^{*}=\arg \max \left(\frac{R_{k, n}^{s}(t)}{T h^{s} k, n(t)}\right) ; k=1, \ldots, K$

where $R_{k, n}^{S}(t)$ is the estimated instantaneous data rate $T h^{s} k, n(t)$ the average throughput.

The updated for scheduling as in (15),

$T h^{s} k, n(t+1)=\left\{\begin{array}{c}\left(1+\frac{1}{t_{c}}\right) T h^{s} k, n(t)+\frac{1}{t_{c}} R_{k, n}^{s}(t) ; k=k * \\ \left(1+\frac{1}{t_{c}}\right) T h^{s}{ }_{k, n}(t) ; k \neq k *\end{array}\right.$

\subsection{Overview of PSO Algorithm}

A Particle Swarm Optimization algorithm is a stochastic optimization technique, based on the population, was proposed by Kennedy and Eberhart, inspired by behavior of bird flocking and fish schooling [26], looking for an optimal solution to the problem posed. Employing a whole of solutions potentials called Particles. Two factors characterize each particle its position and its velocity. Throughout successive iterations, each particle updates its position using its historical best position (pbest) in sight to reach the global best position (gbest) according to the following equations [27]:

$V_{j}($ iter +1$)=Z\left(W^{*} V_{j}(\right.$ iter $)+c_{1} r_{1}\left(\right.$ lpbest $p a r-C_{j}($ iter $\left.)\right)$
$+c_{2} r_{2}{ }^{\text {(gbest }}$ par $-C_{j}($ iter $\left.\left.)\right)\right)$

$C_{j}($ iter +1$)=C_{j}($ iter $)+V_{j}($ iter +1$)$

where $j$ is the number of particles. iter is the iteration number. $Z$ is construction factor; $w$ is inertia weight; $c_{1}, c_{2}$ are the coefficient acceleration; $r_{1}, r_{2}$ are random numbers uniformly distributed in the range $[0,1]$.

The updating process is essentially based on three components. The first term $\mathrm{v}_{\mathrm{j}}^{\text {iter }}$ is the particle 
previous velocity; it serves as memory of the previous flight direction preventing particle from drastically changes of its direction. The second term $\left(c_{1} * r_{1} *\left(\operatorname{pbest}(j)-C_{j}^{i t e r}\right)\right)$ is personal experience of the particle also called cognitive component. The effect of this term is that particle is drawn back to its own best position, modeling its tendency to return to places that were most satisfying on the past. The third term $\left(c_{2} * r_{2} *\left(\right.\right.$ gbest $\left.\left.-C_{j}^{i t e r}\right)\right)$ represents the cooperation among particles, also named social component. The effect of this term is that each particle is also drawn towards the best position found by its neighbor [28].

\subsection{Hybridation PFS-PSO-Based Resource Allocation Algorithm}

For the optimal allocation of resources, the base station collects mobile location information, if users in a cell sectoral are distributed in critiques locations with a low SINR cannot obtain a good quality of service. The idea in this paper, we propose a new resource allocation program in a trisectoral cell based on location of mobiles, channel conditions of users and their demands. This new program combines an evolutionary algorithm and the proportional fair scheduling. Hybridization between PSO and PFS provides a favorable sharing of resources for users in critical locations. However, the particle swarm optimization (PSO) policy is adapted to improve the performances of proportional fairness which is incorporated a set of constraints into the optimization problem.

The process of Resource Allocation scheme using Hybridation PSO and PFS algorithm operates as follows:

First, we generate the resource allocation matrix by using Algorithm 1 which is defined as the vector of $\mathrm{j}$-th particle.

$$
C_{j}=\left\{b_{j 1}, b_{j 2}, \ldots \ldots b_{j N}\right\}, j=1, \ldots, J .
$$

Second, the velocity of $\mathrm{j}$-th particles $V_{j}$ is defined as the change of the particle positions.

Once the first population is generated, fitness evaluation is performed. The fitness function evaluation to be optimized used in the particle swarm optimization algorithm is the objective function given by Equation (10)

After updating the particles, the fitness function evaluate the new swarm is according as follows

$$
f\left(C_{j}^{i t e r+1}\right)=\delta_{j}^{i t e r+1}
$$

Update the pbest for each particle is calculated:

$$
\text { lpbest }_{j}^{\text {ietr }+1}=\text { lpbest }_{j}^{\text {iter }}+f, \text { lpbest }_{j}^{\text {iter }} \geq f\left(C_{j}^{\text {iter }}\right)
$$

Update de gbest represent the maximal fitness:

$$
\text { gbest }^{\text {iter }+1}=\max \left\{f\left(\text { lpbest }_{j}^{i \text { ter }+1}\right)\right\}
$$

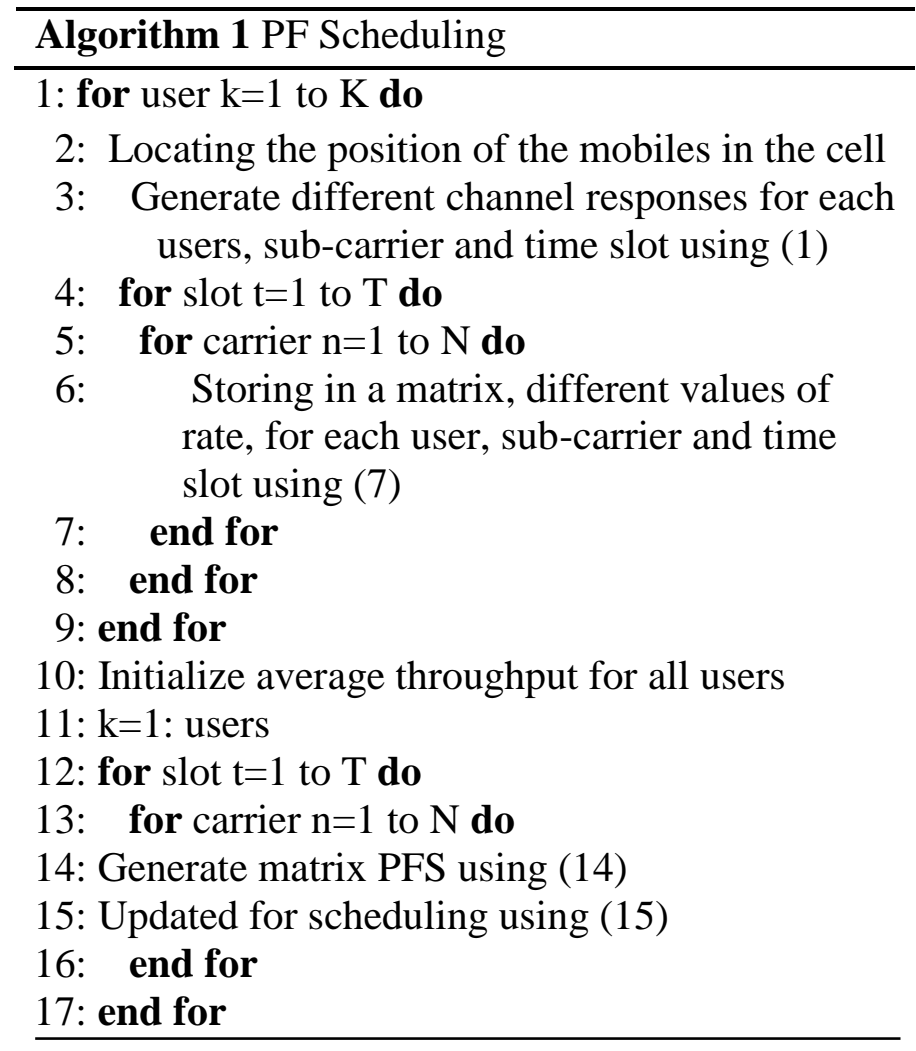


The proposed solution of the resource allocation using the Hybridation PSO-PFS is described in Algorithm 2. The swarm size is a dependent problem and generally kept in between 20 and 50 [29]. The positions and velocities of particles are updated until the number of iteration is achieved; the maximum number of iteration is set to 100 according to the constraints posed. In this problem, the acceleration coefficients and construction factor are set to 1 and 0.7 respectively, at each iteration the inertia weight is calculated as follows $w=\left(\max \_\right.$iterations - iter $) / \max \_$iterations

\section{Algorithm 2 RA- Hybrid PSO-PFS}

1: Initialize max_iterations

2: Initialize swarm and velocities

3: Evaluate initial population, personal best position and global best position

\section{4: Star iterations}

5: while iter $<\max$ _iterations do

6: iter=iter+1

7: for each particle $\mathrm{j}$ do

8: Generate PFS algorithm

9: Calculate the fitness value according to

\section{0: end for}

11: Update the velocity $V_{j}^{\text {iter }}$ using (16)

12: Update particle position using (17)

13: Update the best local position and global best for each particle according to the fitness value

14: end while

15: Output gbest

16: Compute the system performances for each users using $(12,13)$

\section{Simulation and Results}

In this section, we present the evaluation of the proposed algorithms. The system-level parameters are summarized in table 1; we have designed a simulation area of $1 \mathrm{~km} \times 1 \mathrm{~km}$. We assume three types of services, video, voice and transmit data. Furthermore, the approach studied must satisfy a high quality of service, namely a very high throughput, fairness between users and delay constraint. However, a comparison between the proposed schemes is analyzed.

Table 1: Simulation parameters

\begin{tabular}{ll}
\hline Parameters & Values \\
\hline Channel Bandwidth & $20 \mathrm{MHz}$ \\
Carrier Frequency & $2.4 \mathrm{Ghz}$ \\
Number of subcarrier & 1200 \\
Grid layout & $1 \mathrm{cell}$ hexagonal \\
Cell radius & $1 \mathrm{~km}$ \\
BS Transmitter Power & $43 \mathrm{dBm}$ \\
Slot duration & $0.5 \mathrm{~ms}$ \\
Subcarrier Modulation & $16 \mathrm{QAM}$ \\
Number of users & 30 \\
Swarm size of PSO & 50 \\
\hline
\end{tabular}

Figure 2: Positions of all users in the cell

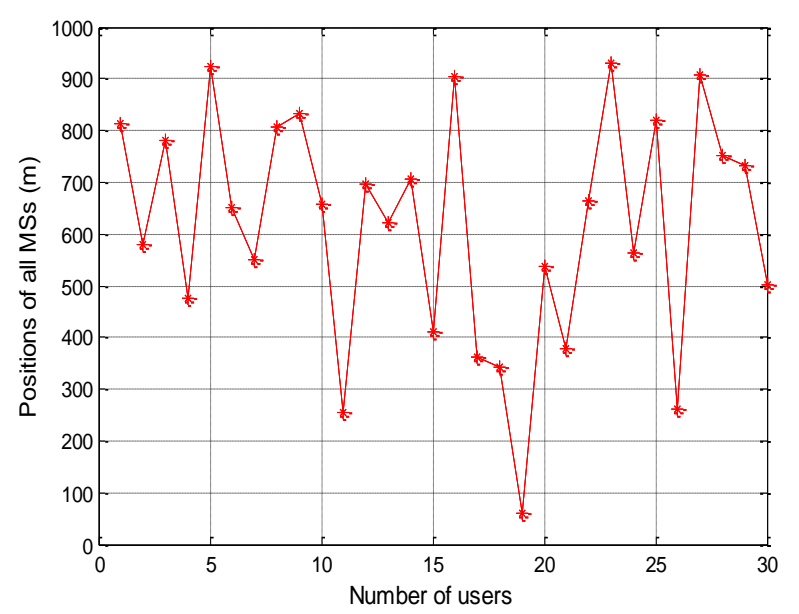

In a tri-sectoral $5 \mathrm{G}$ cell, for each sector the users can have different throughput. In this scenario, at the cell level the reuse of frequency 1 is applied, the users are uniformly distributed as well as the service type; Figure 2 shows the position of each user in the cell. In addition, users with critical positions suffer interference and cause high delays and user's dissatisfaction. In this context, the PFS algorithm takes into consideration the users channel conditions and the PSO is a tool to solve the allocation problem. Figure 3 illustrates the utility of all users and the convergence of the PSO for a number of iterations is 100 , this confirms that the PSO is an algorithm capable of solving the 
allocation problem and improving the scheduler performance.

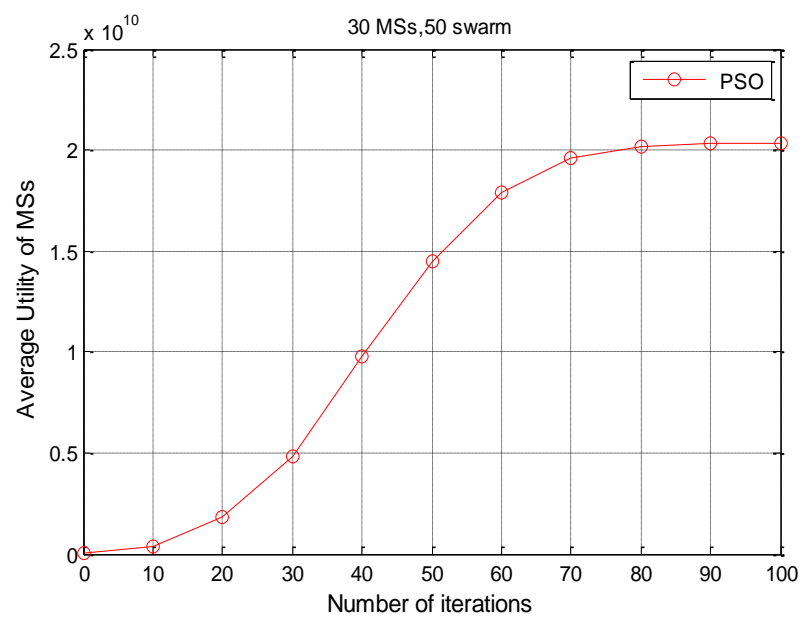

Figure 3: Convergence of PSO iterative algorithm

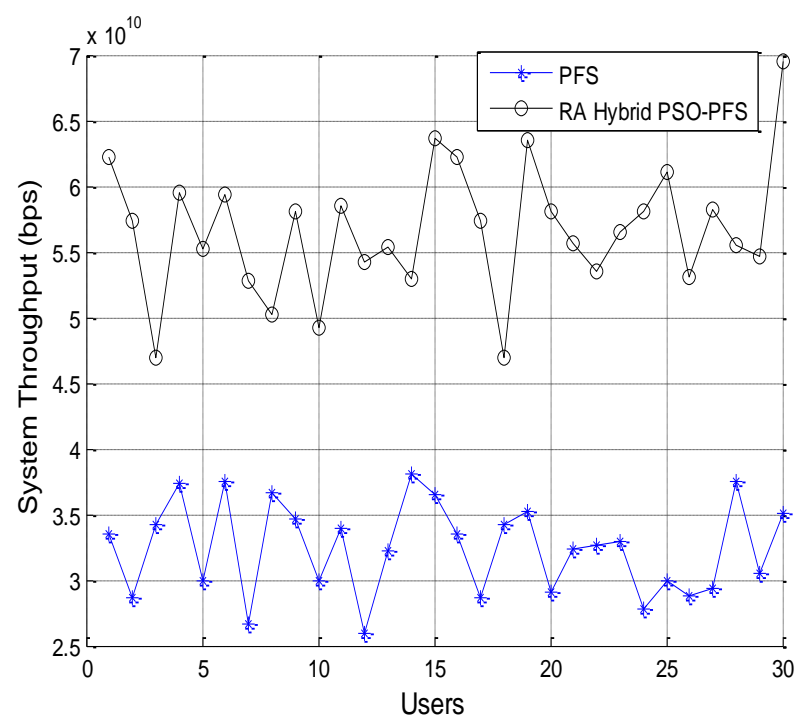

Figure 4: Throughput performance

We had a look at the resource allocation for each user taking into account their channel conditions using the PFS algorithm and the resource allocation with PSO-PFS hybridization, as we can see in Figure 4, a random flow distribution is made taking into consideration the user's position and channel status of each user, the throughput performance of the RA hybrid PSO-PFS is higher than that of the PFS, due to the particularity of the
PSO algorithm, which makes maximum use of the research space, which allows convergence and achieves higher system throughput.

Figure 5 shows the Fairness performance as a function of the number of users. As it can be seen that the equity index is between 0.9 and 1 , it is shown that the allocation by hybrid PSO-PFS ensures fairness between the users.

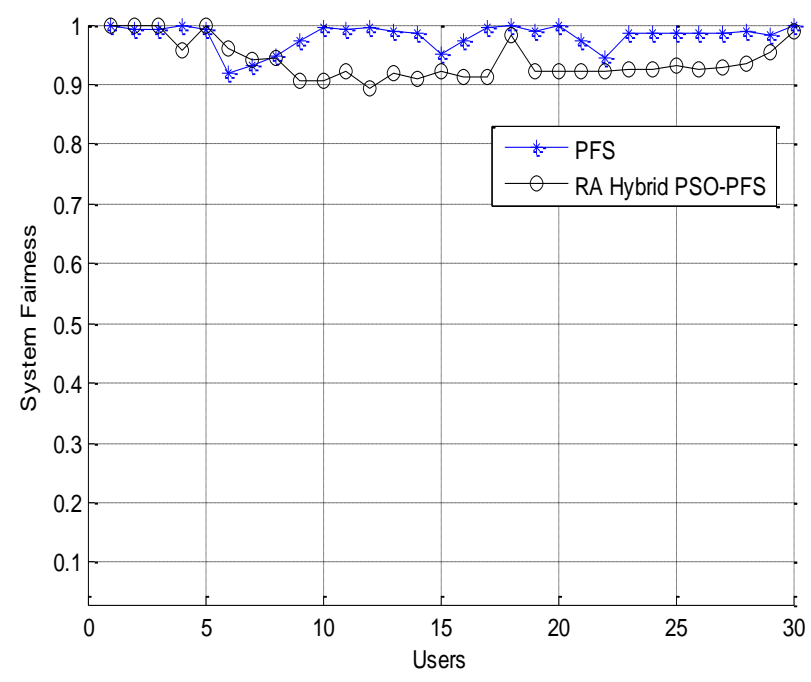

Figure 5: Fairness performance

Concerning the delay constraint, if we compare the two proposed schemes, the simulation results give us an approximation of the number of users that can be served; namely, the larger the number of users in the cell, the delay constraint is important, in that case, with zero delay probability the hybrid PSO-PFS algorithm can serve $60 \%$ compared with the PFS which is $40 \%$ (see figure 6 (a)), as we can also see in the figure 6 (b) the PSO-PFS hybrid algorithm reaches zero probability faster thanks to its convergence towards the optimal solution, which allows it to transmit more resources in each timeslot, and they are sufficient to satisfy the needs of all users with different types of service and according to their position. 


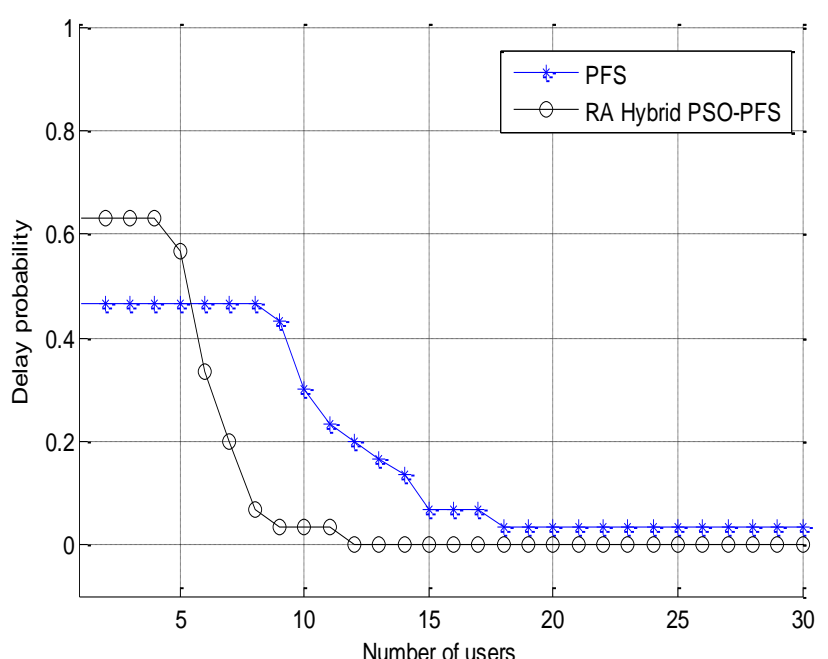

(a) Probability delay as a function of the numbers of users

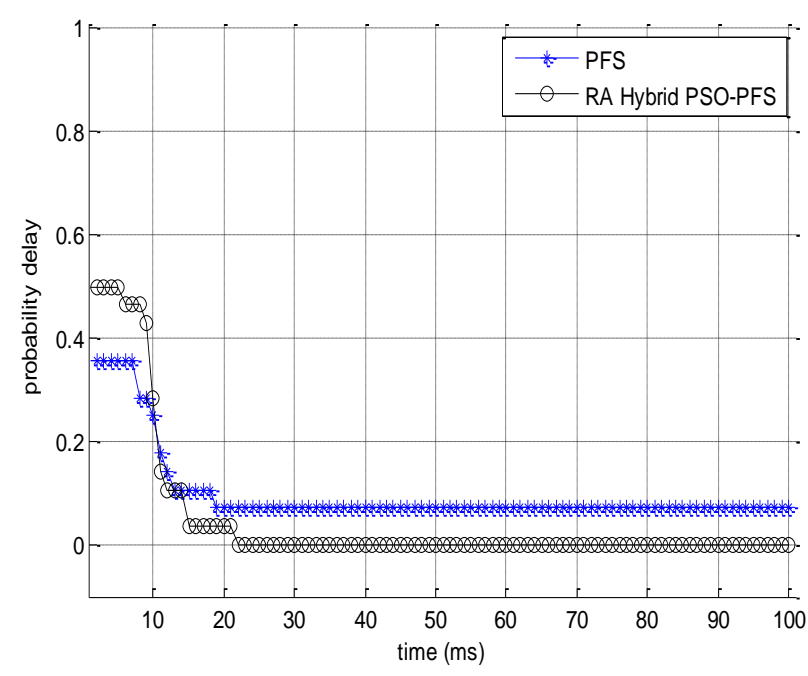

(b) Probability delay as a function of the time

Figure 6: Delay constraint

\section{Conclusion}

In this work, we proposed an optimization approach to solve the resource allocation problem in sectoral cellular networks (4G and 5G system) whose objective is to maximize throughput and ensure users satisfaction. First, we introduced a mathematical model studying the state of channel conditions of each user position. Then, the proposed approach was optimized using the PSOPFS hybrid algorithm, the evolutionary particlebased PSO is adapted to make maximum use of the research space. In addition, our proposed scheme revealed an improvement in the quality of service. Finally, the performance evaluation of the studied system confirms that the PSO-PFS hybridization approach allows a high throughput for all users by ensuring fairness while respecting constraints. However, resource allocation through hybridization could be extended to other approaches such as ensuring a high quality of service for loaded sectors.

\section{References}

[1] Ragaleux. A, Baey. S andGueguen. C. (2015) 'Adaptive and Generic Scheduling scheme for LTE/LTE-A Mobile networks'. Wireless Netw. Springer Science+Business Media. New York.

[2] Gueguen. C, Ezzaouia. M and Yassin. M. (2015) 'Inter-cellular scheduler for $5 \mathrm{G}$ wireless networks'. Physical communication.

[3] D. Parruca., Grysla. M, Gortzenand and S. Gross, J. (2013) 'Analytical Model of Proportional Fair Scheduling in Interference-limited OFDMA/LTE Networks'. IEEE Vehicular Technology Conference.

[4] Qian Li, Rose Qingyang Hu and Yi Qian,Geng Wu. (2013) 'Intracell Cooperation and Resource Allocation in a Heterogeneous Network Relays'. IEEE transactions on vehicular technology vol. 62, No. 4, pp.1770- 1784.

[5] Nsiri. B, Mallouki. N, Mhatli. S, Ghanbarisabagh. M, Ammar. M. Hakimi, W. (2015) 'Modeling and Performance Evaluation of Novel Scheduling Algorithm for Downlink LTE Cellular Network'. Wireless Pers Commun. pp. 2303-2316.

[6] Ankit Saxenal, Sindal. R. (2016) 'Strategy for Ressource Allocation in LTE-A' International Conference on Signal Processing, communication, Power and Embedded system.

[7] Ezzaouia. M, Gueguen. C, Ammar. M, Baey. S, Lagrange. X and Bouallègue. A. (2017) 'A Dynamic Inter-cellular Bandwidth Fair Sharing scheduler for future wireless networks'. Physical communication.

[8] H. Marshoud. H, Otrok. H and Barada. H. (2013) 'Macrocellfemtocells resource allocation with hybrid access motivational model'. Physical communication.

[9] Li. W, Lei. J, Wang. T, Xiong. C and Wei. J. (2016) 'Dynamic Optimization for Resource Allocation in Relay-Aided OFDMA Systems Under Multiservice'. IEEE transactions on vehicular technology vol. 65, No. 3, pp.1303-1313. 
[10] Sun. S, Kim. K and Shin. O. (2015) 'Device-to devise ressource allocation in LTE-advanced networks by hybrid particle swarm optimoization and genetic algorithm'. Springer Science+Business Media.

[11] Lei. H, Zhang. L, Zhang. X and Yang. D. (2007) 'A nouvel Multi-cell OFDMA system structure using fractional frequency reuse'. The $18^{\text {th }}$ Annual IEEE International Symposium on Personal, Indoor and Mobile Radio Communications.

[12] Alitaleshi. A, Ghazizadeh. R and Kalbkhani. H. (2015) 'Ameliorated Ressource Allocation in Two-tier FemtocellMacrocell Networks with Six Directional Antennas for Macrocells'. Springer Science+Business Media.

[13] Claussen. H and ho. L. (2012). 'Multi-carrier Cell Structures with angular Offset'. IEEE $23^{\text {rd }}$ International Symposium on Personal, Indoor and Mobile radio communications.

[14] Pirinen. O. 'A brief overview of 5G Research Activities'. Centre for Wireless Communication, P.O. Box 4500, FI-90014 University of Oulu, Finland.

[15] 3GPP TR 36.814 V9.0.0, (2010) 'Further advancements for E UTRA physical layer aspects' Tech. Rep.

[16] Yilmaz. O.N.C, Hämäläinen. S and Hämäläinen. (2009) 'System level analysis of vertical sectorization for 3GPP LTE', IEEE 6th International Symposium on Wireless Communication System, pp.453-457.

[17] Goldsmith. A. (2005) 'Wireless Communications', Cambridge, U. K. University of Cambridg. Press, vol. 3.

[18] 3GPP TR 36.814 V9.0.0. (2010) 'Further advancements for EUTRA physical layer aspects' Tech. Rep.

[19] Proakis. J and G. Salehi. M. (2008) 'Digital communications'. 5th ed. New York. McGraw Hill.

[20] Kelly. F. Maulloo, A and Tan D. (1998) 'Rate control in communication networks: shadow prices, proportional fairness and stability'. J. Oper.Res. Soc., vol. 49, pp. 237-252.

[21] Yang. S.C. (2010) 'OFDMA System Analysis and Design'.

[22] Parruca. D and Gross.J. (2016) 'Throughput Analysis of Proportional Fair Scheduling for Sparse and Ultra-Dense Interference-Limited OFDMA/LTE Networks' IEEE Transactions on Wereless Communications, vol.10, No.15, pp. 6857-6870.

[23] Zeandji. S, Gueguen. C, Ouamri. A and Khireddine. A. (2018) 'Resource allocation in a multi-carrier cell using scheduler algorithms'. In proc. IEEE $4^{\text {th }}$ International Conference on Optimization and Applications.

[24] Viswanath. P, Tse. D. N. C and Laroia R. (2002) 'Opportunistic beamforming using dumb antennas' IEEE Trans. Inf. Theory, vol. 48, no. 5, pp. 1277-1294.

[25] Batista. R.L, Silva. CF.M.e, Da Silva Jr. JMB, Maciel. TF. and Cavalcanti. FR.P. (2014) 'What happens with a proportional fair cellular scheduling when D2D communications underlay a celluler network?'. IEEE WCNC - Workshop on Device-toDevice and Public Safety communications, pp 260-265,

[26] Kennedy. J and Eberhart. R.C. (1995). 'Particle swarm optimization'. In Proc. IEEE International Conference Neural Network Piscataway, NJ, USA, pp. 1-5.

[27] Brikh. L, Guenounou. O, Yahiaoui. F, Kacimi M. A and Ouaret A.. (2016) 'Optimization of TSK Fuzzy Model Using New Improved PSO' International Science Press. IJCTA. pp 323333.

[28] Eslami. M, Shareef. H, Khajehzadeh M and Mohamed. A. (2012) 'Asurvey of the state of the art in Particle Swarm Optimization'. Research journal of applied Sciences, Engeneering and technology 4(9), pp1181-1197
[29] Eberhart. R and Kennedy. J. (1995) 'A new optimizer using particle swarm theory'. IEEE, In: Sixth International Symposium on Micro Machine and Humain Science, , pp. 3944.

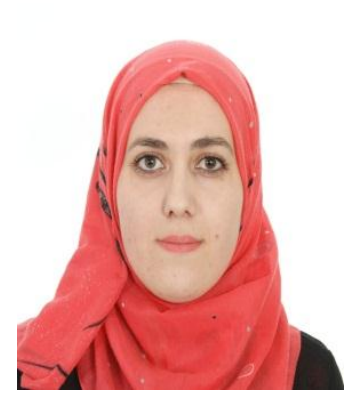

Zenadji Sylia is currently a $\mathrm{PhD}$ candidate in Wireless mobile communication at Department of Electrical Engineering, University of Bejaia, Algeria. His research interests are resource allocation in LTE network, cellular network planning, OFMD, Scheduling Algorithms and Meta-heuristic Algorithms.

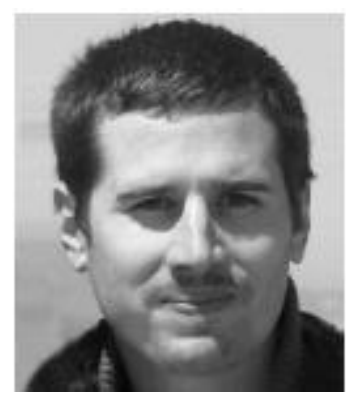

Since September of 2011, Cédric Gueguen is associate professor at University of Rennes 1 and member of ATNET team at IRISA Labs. His main research interests are related to wireless network and particularly to scheduling algorithms and green networking. Basically, he teaches on performance evaluation, Internet protocol and routing. His Ph.D. thesis focused on the efficient support of multimedia services in OFDM wireless networks, both in the uplink and in the downlink. New access schemes were studied which further increases the benefits of opportunistic scheduling mainly by extending this cross layer technique to higher layers.

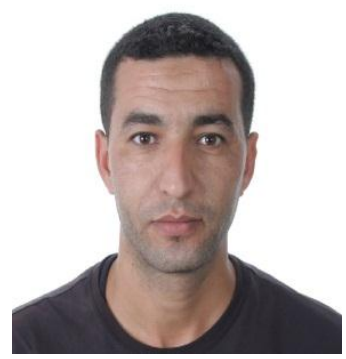

Brikh Lamine is currently a $\mathrm{PhD}$ candidate in Automatic at Department of Electrical Engineering, University of Bejaia, Algeria. His research interests Optimization of TSK fuzzy model using PSO algorithm and Metaheuristic Algorithms. 


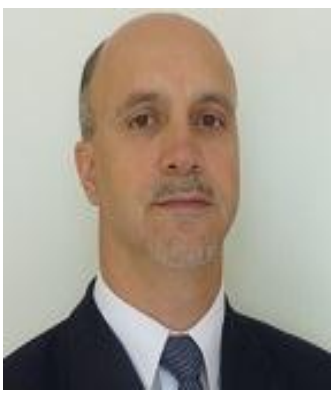

Talbi Larbi is currently Professor and Head of the Department of Computer and Engineering University of Quebec in Outaouais (UQO), Gatineau, Canada. His main research interests are related to wireless communication and antenna propagation and MIMO. In 2013, he was awarded the Best Paper Prize of IET - ICWCA conference held in Malaysia. Currently he is actively involved in major projects related to the deployment of wireless technologies in underground mines, mainly, experimental characterization of the underground mine channels using MIMO antennas at $60 \mathrm{GHz}$, design of microwave and $\mathrm{RF}$ components using SIW technique, transparent antennas, metamaterials applied to microwave design, antenna array for wireless applications.

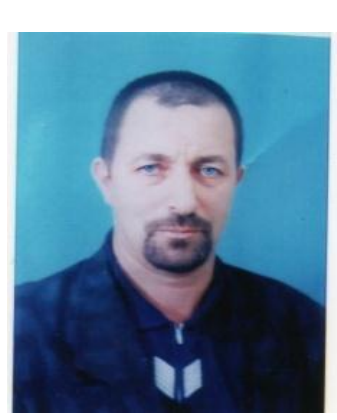

Abdelkrim Khireddine
received his BS in
Mathematics in 1978 from
University of Constantine, an
Engineering degree in
Telecommunication from the
University of Oran with
honours in 1981 and MSc in
Electronics from the University of Setif in Algeria in 1994, a PhD in Communication from the University of Setif in 2006. He began work at the telecommunication minister in 1981. He was selected as a Supervisor in 1983, and later joined the Division Communication Bejaia Center. $\mathrm{He}$ was the president of electronic doctoral school in Bejaia University and expert doctoral thesis and academic accreditation (2009-2017). His research interests include planning and optimization of mobile networks, satellite communication, and antenna modeling. 